\title{
NO-assisted molecular-beam epitaxial growth of nitrogen substituted EuO
}

\author{
R. Wicks, ${ }^{1}$ S. G. Altendorf, ${ }^{2,}$ a) C. Caspers, ${ }^{2, \text { b) }}$ H. Kierspel, ${ }^{2}$ R. Sutarto, ${ }^{2, c)}$ L.H. Tjeng, ${ }^{2,3, \text { d) }}$ and A. \\ Damascelli ${ }^{1,4, e)}$ \\ ${ }^{1)}$ Department of Physics and Astronomy, University of British Columbia, Vancouver, British Columbia V6T 1Z1, \\ Canada \\ ${ }^{2)}$ II. Physikalisches Institut, Universität zu Köln, Zülpicher Str. 77, 50937 Köln, Germany \\ ${ }^{3)}$ Max Planck Institute for Chemical Physics of Solids, 01187 Dresden, Germany \\ 4) Quantum Matter Institute, University of British Columbia, Vancouver, British Columbia V6T 1Z4, \\ Canada
}

(Dated: 15 June 2021)

\begin{abstract}
We have investigated a method for substituting oxygen with nitrogen in EuO thin films, which is based on molecular beam epitaxy distillation with NO gas as the oxidizer. By varying the NO gas pressure, we produce crystalline, epitaxial $\mathrm{EuO}_{1-\mathrm{x}} \mathrm{N}_{\mathrm{x}}$ films with good control over the films' nitrogen concentration. In-situ x-ray photoemission spectroscopy reveals that nitrogen substitution is connected to the formation $\mathrm{Eu}^{3+} 4 f^{6}$ and a corresponding decrease in the number of $\mathrm{Eu}^{2+} 4 f^{7}$, indicating that nitrogen is being incorporated in its 3- oxidation state. While small amounts of $\mathrm{Eu}^{3+}$ in over-oxidized $\mathrm{Eu}_{1-\delta} \mathrm{O}$ thin films lead to a drastic suppression of the ferromagnetism, the formation of $\mathrm{Eu}^{3+}$ in $\mathrm{EuO}_{1-\mathrm{x}} \mathrm{N}_{\mathrm{x}}$ still allows the ferromagnetic phase to exist with an unaffected $\mathrm{T}_{\mathrm{c}}$, thus providing an ideal model system to study the interplay between the magnetic $f^{7}(\mathrm{~J}=7 / 2)$ and the non-magnetic $f^{6}(\mathrm{~J}=0)$ states close to the Fermi level.
\end{abstract}

The key to producing novel spintronic devices is to find magnetic materials that can be combined with conventional semiconductors. One class of materials being considered is that of the dilute magnetic semiconductors (DMS). In this case, standard semiconductors are doped with magnetic impurities, leading to spin-dependent electron transport phenomena which stem from the conduction-electron-mediated ferromagnetic coupling ${ }^{1,2}$. Another promising class of materials is that of the ferromagnetic semiconductors (FMS) from the rare earth pnictide and chalcogenide families. Despite currently having lower Curie temperatures $\left(T_{c}\right)$ than the DMSs, the rare earth compounds are interesting because of their spectacular properties due to the interplay between the large magnetic moments arising from the atomic-like forbitals and the electrons in the wide conduction bands. Arguably the archetypical FMS is EuO, with its $\mathrm{J}=7 / 2$ moment from the $\mathrm{Eu}^{2+} f^{7}$ ions, a $\mathrm{T}_{\mathrm{c}}$ of $69 \mathrm{~K}$, with a spin-split conduction band which allows for spin tunneling with up to $100 \%$ spin polarization ${ }^{3-5}$. It exhibits a metal-insulator transition as a function of applied magnetic field and temperature with a resistivity jump of 6 and 8 orders of magnitude, respectively ${ }^{6-8}$; this is even higher than what is observed in the colossal magneto-resistance manganites ${ }^{9,10}$. Furthermore, $\mathrm{EuO}$ was grown successfully on $\mathrm{Si}, \mathrm{GaN}$ and $\mathrm{GaAs}^{4,11-13}$, and could thus be readily incorporated in conventional semiconductor technology. Similarly GdN, which is isoelectronic and isostructural to EuO, exhibits many of the same interesting properties with a $\mathrm{T}_{\mathrm{c}}$ of about $60 \mathrm{~K}^{14,15}$.

\footnotetext{
a) Present address: Max Planck Institute for Chemical Physics of Solids, Nöthnitzer Str. 40, 01187 Dresden, Germany

${ }^{b)}$ Present address: Peter Grünberg Institut (PGI-6), Forschungszentrum Jülich, 52425 Jülich, Germany

${ }^{c}$ Present address: Canadian Light Source, University of Saskatchewan, Saskatoon, Saskatchewan S7N 0X4, Canada

d) Electronic mail: Hao.Tjeng@cpfs.mpg.de

e)Electronic mail: damascelli@physics.ubc.ca
}

In the search for new compounds in the area of FMSs, and based on the promising properties of $\mathrm{EuO}$ and $\mathrm{GdN}$, another system that has attracted some attention is EuN. At first glance this might appear surprising, due to the non-magnetic, $\mathrm{J}=0$ character of its $\mathrm{Eu}^{3+} f^{6}$ ions. However, recent band structure calculations seem to indicate that the $\mathrm{J}=0$ state can be spin polarized, giving rise to ferromagnetism with an unoccupied $f$ band located close to - or even right at - the chemical potential $^{16-18}$. This would lead to the realization of halfmetallic ferromagnetic behavior. Ruck et al. have investigated this possibility in EuN films grown by molecular beam epitaxy (MBE). However, by studying the magnetic properties by Xray magnetic circular dichroism, they found no evidence for a ferromagnetic state ${ }^{19}$.

In this work, in search of half-metallic ferromagnetic behavior associated with nominally non-magnetic $\mathrm{Eu}^{3+}$ ions, we follow a different approach. Rather than pure EuN, we grow thin films of $\mathrm{EuO}_{1-x} \mathrm{~N}_{x}$ aiming at embedding the $\mathrm{Eu}^{3+} f^{6}$ ions induced by nitrogen substitution in the welldefined ferromagnetic lattice of EuO. This is in accordance with the above-mentioned rationale that the magnetization provided by the EuO lattice on non-magnetic $\mathrm{Eu}^{3+} f^{6}$ might lead to the formation of a spin-polarized conduction band, whose filling is directly controlled by the nitrogen concentration. As a result of the narrow bandwidth of the $f$-derived band, these carriers would have by a large effective mass.

Since $\mathrm{EuO}_{1-\mathrm{x}} \mathrm{N}_{\mathrm{x}}$ has not been synthesized before, we must first determine if such a system can be grown. It is widely known that it is difficult to grow pure EuO thin films. EuO is extremely unstable in air, and even in UHV environment - if it is grown with too much supply of oxygen - it will form $\mathrm{Eu}_{2} \mathrm{O}_{3}$ and/or $\mathrm{Eu}_{3} \mathrm{O}_{4}$ phases. On the other hand, if it is grown with too little supply of oxygen, Eu metal clusters may form ${ }^{20}$. Both of these situations will deteriorate the extraordinary properties of EuO. These problems can be overcome to successfully grow high-quality $\mathrm{EuO}$ thin films by the MBE distillation method, which involves evaporating europium metal onto a hot substrate in a low pressure of 


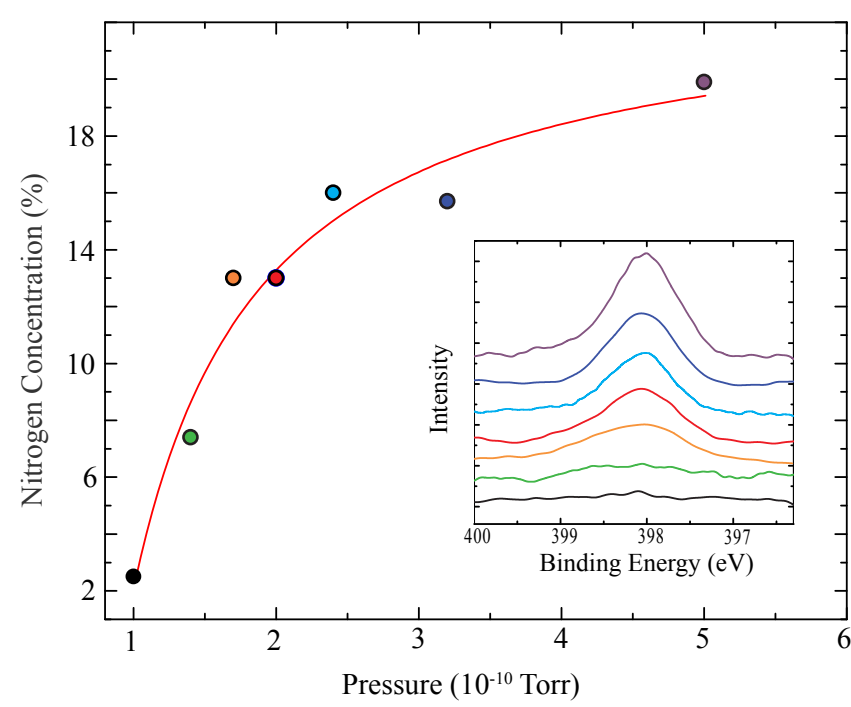

FIG. 1. Increase in nitrogen concentration in the $\mathrm{EuO}_{1-\mathrm{x}} \mathrm{N}_{\mathrm{x}}$ films as a function of $\mathrm{NO}$ partial pressure. These concentrations were measured using the ratio of the background-subtracted nitrogen and oxygen $1 s$ core level areas in XPS, corrected for their corresponding photo-ionization cross-sections. The inset shows the increase in the nitrogen $1 s$ core level peak with increasing NO pressure as measured by XPS. The red line is a guide to the eye, based on a fit to the Langmuir adsorption equation, see Ref. 26.

oxygen $^{3,21-23}$. The low oxygen pressure prevents the formation of $\mathrm{Eu}_{2} \mathrm{O}_{3}$ and/or $\mathrm{Eu}_{3} \mathrm{O}_{4}$, while any unreacted metal is reevaporated from the hot substrate surface thus maintaining the well-proven europium distillation growth technique ${ }^{23-25}$. This is the approach we have chosen to grow our $\mathrm{EuO}_{1-\mathrm{x}} \mathrm{N}_{\mathrm{x}}$ films; however, instead of pure oxygen, we used NO gas, and during the course of the work we also discovered that the oxygennitrogen substitution can be tuned.

The choice of NO gas as both the oxidizer and as a means of substituting nitrogen in $\mathrm{EuO}$ was inspired by earlier $\mathrm{NO}_{2}$ assisted epitaxial growth of $\mathrm{Fe}_{3} \mathrm{O}_{4}, \mathrm{Fe}_{1-\delta} \mathrm{O}$, and $\mathrm{CrO}^{27,28}$. There, $\mathrm{NO}_{2}$ gas was used because it is a very efficient oxidizer; as a side effect it was found that, in addition to the desired amount of oxygen, nitrogen was also being incorporated into the films. Since the nitrogen concentration in the films was decreasing upon increasing $\mathrm{NO}_{2}$ pressure, it was hypothesized that the probability of nitrogen substitution was higher when there was insufficient oxygen to form a stoichiometric material. Since the conditions in MBE distillation are always oxygen deficient by design, this technique can be used as a general approach to incorporate nitrogen into oxide films. For the case of $\mathrm{EuO}$, since $\mathrm{NO}_{2}$ is far too aggressive an oxidizer, we chose NO gas instead, following the work on nitrogen substituted $\mathrm{SrO}$ by Elfimov et al. ${ }^{29}$. In that work, by keeping the rate of metal evaporation constant, and changing the background pressure of NO gas in the UHV growth chamber, it was shown that the amount of nitrogen taken up by the film can be tuned.

The $\mathrm{EuO}_{1-\mathrm{x}} \mathrm{N}_{\mathrm{x}}$ samples were grown on yttria-stabilized zirconia (YSZ) substrates, whose $5.142 \AA$ lattice constant is very

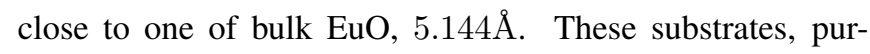
chased from SurfaceNet $\mathrm{GmbH}$., were annealed in the growth chamber for two hours at $600^{\circ} \mathrm{C}$ in $1 \times 10^{-6}$ Torr of oxygen (the chamber base pressure is in the $10^{-10}$ Torr range). This procedure removes surface contaminants, re-oxygenates the substrate, and gives defects on the surface enough mobility to aggregate into step edges, producing an atomically flat surface. After annealing, the substrate temperature was set to $450^{\circ} \mathrm{C}$. Europium metal was evaporated from a Knudsen cell at a rate of $8.2 \AA$ per minute; the rate was measured with a quartz crystal monitor. The chamber was backfilled with NO gas through a precision leak valve and the NO partial pressure was measured with a MKS Instruments residual gas analyzer. While Eu evaporation rate and substrate temperature were kept constant for all growths, the amount of NO gas used to oxidize and dope the films was adjusted for each growth. The range of gas pressures was between $1 \times 10^{-10}$ and $5 \times 10^{-10}$ Torr. The choice of substrate temperature, evaporation rate, and NO pressure range determine if the conditions are favourable for MBE distillation; starting parameters were chosen based on earlier work ${ }^{23,30}$.

Fig. 1 demonstrates how the amount of nitrogen incorporated into the $\mathrm{EuO}_{1-\mathrm{x}} \mathrm{N}_{\mathrm{x}}$ films varies by changing the background pressure of the NO gas. The concentration of substituted nitrogen was estimated from the photo-ionization cross-section $^{31}$ corrected ratio of nitrogen and oxygen $1 s$ core

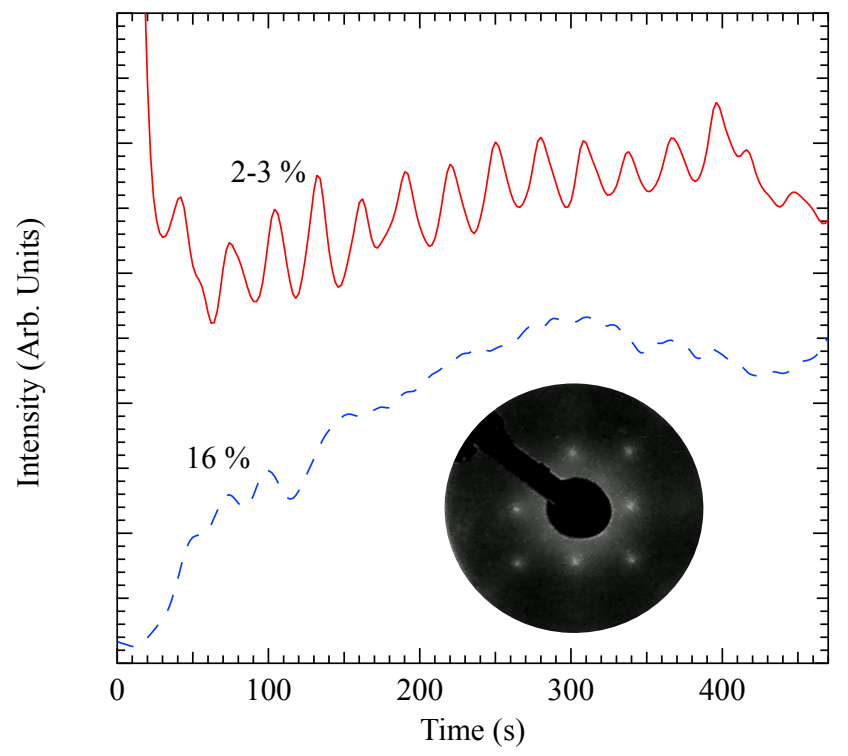

FIG. 2. RHEED spot intensity oscillations for $2-3 \%$ and $16 \%$ nitrogen concentration, respectively. The inset shows a LEED diffraction pattern for a $13 \% \mathrm{~N}$ substituted sample. The initial three oscillations in the $16 \%$ nitrogen substitution case are consistent with a layer-by-layer growth process enabled by oxygen being donated to the film by the YSZ substrate. The $2-3 \%$ substitution film exhibits in addition RHEED oscillations that continue well past the thickness where oxygen donation from the substrate could have an effect, indicating that these prolonged oscillations are due to sustained layer-bylayer growth of $\mathrm{EuO}_{1-\mathrm{x}} \mathrm{N}_{\mathrm{x}}$. The LEED diffraction pattern indicates that even after 100 minutes of growth, the film remains crystalline. 


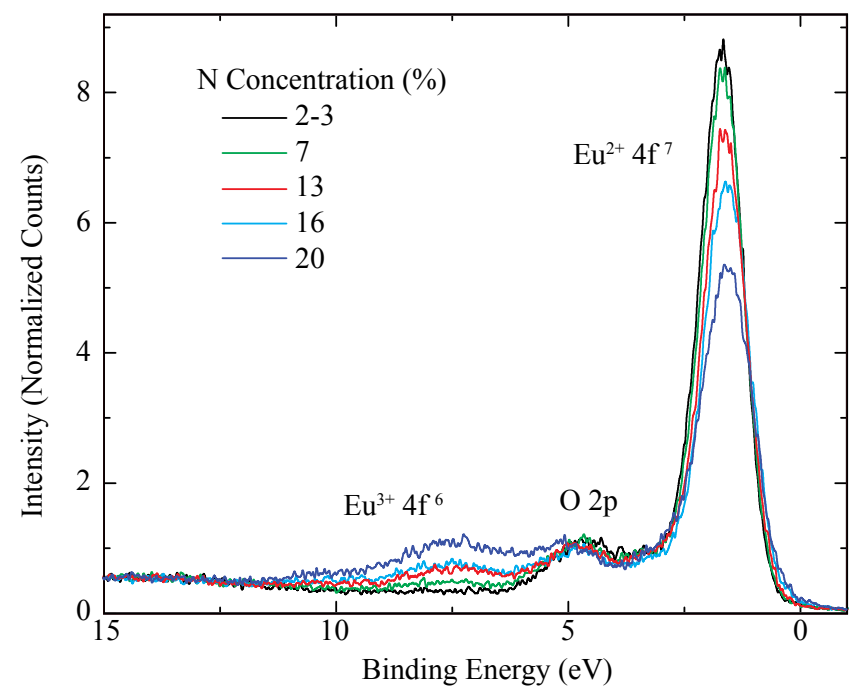

FIG. 3. Valence band XPS spectra of $\mathrm{EuO}_{1-\mathrm{x}} \mathrm{N}_{\mathrm{x}}$ as a function of nitrogen concentration. The increase of spectral intensity in the $\mathrm{Eu}^{3+}$ peak, and the corresponding decrease in $\mathrm{Eu}^{2+}$ spectral weight, suggest that nitrogen is being incorporated in its $3^{-}$oxidation state. The XPS spectra were normalized to the total number of counts.

level peaks measured by x-ray photoemission spectroscopy $(\mathrm{XPS})^{28}$. The XPS measurements were performed in situ with monochromatized $\mathrm{Al} K \alpha$ radiation and a VSW 150 electron analyzer. As shown in the inset of Fig. 1, the nitrogen $1 s$ peak grows with the NO pressure (the spectra were normalized to the oxygen $l s$ peak area, not shown).

We note that the non-linear increase of nitrogen concentration into the $\mathrm{EuO}_{1-\mathrm{x}} \mathrm{N}_{\mathrm{x}}$ films with increasing NO pressure is very different from the previously reported linear decrease with increasing gas pressure ${ }^{27,28}$, observed in the $\mathrm{NO}_{2}$ assisted growth of $\mathrm{Fe}_{3} \mathrm{O}_{4}, \mathrm{Fe}_{1-\delta} \mathrm{O}$, and $\mathrm{CrO}$. In these latter cases, the metal-to- $\mathrm{NO}_{2}$ flux ratio was approximately 1 , setting the growth far outside of the MBE distillation regime used here. One could then envision that the $\mathrm{NO}_{2}$ oxidizes first the available $\mathrm{Fe}$ or $\mathrm{Cr}$, leaving behind an equal number of NO molecules that can react with the remaining metal; since the number of these remaining metal sites is inversely proportional to the initial $\mathrm{NO}_{2}$ pressure, one obtains the observed linear decrease in nitrogen concentration with increasing $\mathrm{NO}_{2}$ pressure. For the present case of NO-assisted growth of $\mathrm{EuO}$, we can conclude that the increase of the N-to-O ratio with increasing NO pressure stems specifically from the MBE distillation conditions, although further research will be needed for the accurate quantitative modeling of the adsorption kinetics under these conditions.

In addition to providing a method for introducing nitrogen into EuO films, MBE distillation with NO gas also produces films with excellent crystalline quality. All of the films exhibited low-energy electron diffraction (LEED) and reflection high-energy electron diffraction (RHEED) patterns, with well-defined diffraction spots after growth. The films also exhibited layer-by-layer growth under certain conditions, as indicated by the presence of typical RHEED oscillations (see

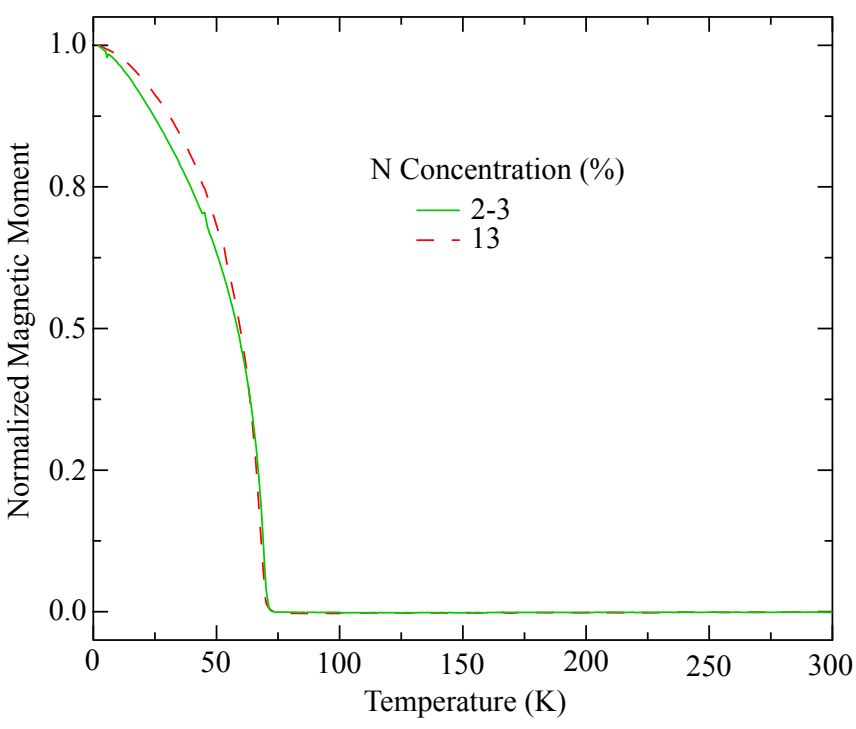

FIG. 4. Magnetization curves for two different $\mathrm{EuO}_{1-\mathrm{x}} \mathrm{N}_{\mathrm{x}}$ samples at 10 Gauss. The transition temperature remains unchanged from the EuO value of $69-70 \mathrm{~K}$, even at high levels of nitrogen substitution.

Fig. 2 for representative RHEED data from $2-3 \%$ and $16 \%$ nitrogen substituted $\mathrm{EuO}_{1-\mathrm{x}} \mathrm{N}_{\mathrm{x}}$, and crystalline LEED data from a $13 \%$ substituted sample). The $16 \%$ substitution level does exhibit three RHEED oscillations at the beginning of growth, but they quickly disappear. These initial RHEED oscillations are seen in all the films, regardless of the substitution level; however, the lower pressure growths exhibit RHEED oscillations that continue for several 10's of monolayers. The difference between high and low pressure regimes is most likely due to a too high concentration of defects in the heavily nitrogen-substituted films; these defects act as nucleation sites, initiating three-dimensional island growth and destroying the two-dimensional, layer-by-layer growth. Initially, however, the growth is primarily controlled by oxygen being donated to the film by the YSZ substrate, rather than by the NO gas ${ }^{23}$ : this allows the observation of layer-by-layer growth independent of the NO gas pressure. RHEED oscillations that continue beyond 4-5 monolayers cannot be due to the substrate donating oxygen, because the film is at that point too thick for oxygen from the substrate to diffuse to the surface $^{23}$. Therefore, these additional oscillations must originate from $\mathrm{EuO}_{1-\mathrm{x}} \mathrm{N}_{\mathrm{x}}$ growing in a truly layer-by-layer mode, as in the case of the $2-3 \%$ substitution level shown in Fig. 2.

The results discussed above demonstrate that MBE distillation with $\mathrm{NO}$ gas is a suitable approach for producing high crystallinity, epitaxial $\mathrm{EuO}_{1-\mathrm{x}} \mathrm{N}_{\mathrm{x}}$ films, with tunable nitrogenoxygen substitution. To establish the oxidation state of the substituted nitrogen, and provide a more detailed characterization of the films' electronic structure, we performed XPS valence band measurements in situ (Fig. 3). The spectra evolution indicates that as the nitrogen concentration increases so does the amount of $\mathrm{Eu}^{3+}$ spectral weight, while the $\mathrm{Eu}^{2+}$ intensity decreases. We also note that the oxygen $2 p$ intensity does not increase with increasing NO-pressure, establishing 
that the increase of $\mathrm{Eu}^{3+}$ is due to the substitution of $\mathrm{O}^{2-}$ with $\mathrm{N}^{3-}$, thus with nitrogen being incorporated in its 3- oxidation state. Whereas these XPS results match those from over-oxidized $\mathrm{Eu}_{1-\delta} \mathrm{O}$ films, the electronic/magnetic properties of $\mathrm{EuO}_{1-\mathrm{x}} \mathrm{N}_{\mathrm{x}}$ are remarkably different. In particular, at variance with the behavior observed for $\mathrm{Eu}_{1-\delta} \mathrm{O}$ where small amounts of $\mathrm{Eu}^{3+}$ lead to a drastic suppression of the ferromagnetism ${ }^{23}$, over-oxidation in $\mathrm{EuO}_{1-\mathrm{x}} \mathrm{N}_{\mathrm{x}}$ still produces a lineshape of the magnetization curve which is Brillouin-like, suggesting an appreciable preservation of the ferromagnetic phase. Important is that the $\mathrm{T}_{\mathrm{c}}$ of about $69 \mathrm{~K}$, as in pure $\mathrm{EuO}$, is observed over a wide range of nitrogen substitution. This is demonstrated by magnetization measurements performed ex situ with a Quantum Designs MPMS-XL7 SQUID magnetometer in a 10 Gauss field (after capping the samples with a thick aluminum layer to protect them from further oxidation when removed from the MBE system), and here shown in Fig. 4.

In conclusion, by substituting nitrogen for oxygen in $\mathrm{EuO}$, we have made a $\mathrm{Eu}^{2+} / \mathrm{Eu}^{3+}$ system that remains ferromagnetic despite the inclusion of $\mathrm{Eu}^{3+} 4 f^{6}$ sites, something not possible in the more extensively studied $\mathrm{Eu}_{1-\delta} \mathrm{O}$. In addition, $\mathrm{EuO}_{1-\mathrm{x}} \mathrm{N}_{\mathrm{x}}$ is also the ideal system for the specific purpose of studying the hopping between the $f^{7}(\mathrm{~J}=7 / 2)$ and $f^{6}$ $(\mathrm{J}=0)$ levels located in proximity of the chemical potential. In this respect, $\mathrm{EuO}_{1-\mathrm{x}} \mathrm{N}_{\mathrm{x}}$ is also better than $\mathrm{Eu}_{x} \mathrm{Gd}_{1-x} \mathrm{~N}-$ in which such $f^{6} / f^{7}$ mixing is also achieved - since in the latter case the $4 f^{7}$ levels of $\mathrm{Eu}$ and $\mathrm{Gd}$ are split in energy by several $\mathrm{eV}$ 's ${ }^{30}$, preventing an efficient hopping within the $f$ band. More generally, the MBE NO-assisted distillation technique described here provides a means to tune the oxygen-nitrogen substitution in other binary oxides.

We thank I.S. Elfimov and G.A. Sawatzky for discussions and suggestions, and L. Hamdan and T. Koethe for technical assistance. We acknowledge support from the Max Plank - UBC Centre for Quantum Materials. The work at UBC was supported by the Killam, Sloan, CRC, NSERC's Steacie Fellowship Programs (A.D.), NSERC, CFI, CIFAR Quantum Materials, and BCSI. The research activities in Cologne were supported by the Deutsche Forschungsgemeinschaft through SFB 608.

${ }^{1}$ T. Dietl, H. Ohno, F. Matsukura, J. Cibert, and D. Ferrand, Science 287, 1019 (2000).

${ }^{2}$ S. J. Pearton, W. H. Heo, M. Ivill, D. P. Notron, and T. Stiener, Semicond. Sci. Technol. 19, R59 (2004).

${ }^{3}$ P. Steeneken, L. Tjeng, I. Elfimov, G. Sawatzky, G.Ghiringhelli, N. Brooks, and D.-J. Huang, Phys. Rev. Lett. 88, 047201 (2002).

${ }^{4}$ A. Schmehl, V. Vaithyanathan, A. Herrnberger, S. Thiel, C. Richter, M. Liberati, T. Heeg, M. Roecherath, L. Kourkoutis, S. Muehlbauer,
P. Boeni, D. Muller, Y. Barash, J. Schubert, Y. Idzerda, J. Mannhart, and D. Schlom, Nat. Mater. 6, 882 (2007).

${ }^{5}$ R. Panguluri, T. Santos, E. Negusse, J.Dvorak, Y.Idzerda, J. Moodera, and B. Nadgoryny, Phys. Rev. B 78, 125307 (2008).

${ }^{6}$ M. Oliver, J. Dimmock, A. McWhorter, and T. Reed, Phys. Rev. B 5, 1078 (1972).

${ }^{7}$ Y. Shapira, S. Foner, and T. Reed, Phys. Rev. B 8, 2299 (1973).

${ }^{8}$ Y. Shapira, S. Foner, R. Aggarwal, and T. Reed, Phys. Rev. B 8, 2316 (1973).

${ }^{9}$ A. Ramirez, J. Phys.: Condens. Matter 9, 8171 (1997).

${ }^{10}$ M. Imada, A. Fujimori, and Y. Tokura, Rev. Mod. Phys. 70, 1039 (1998).

${ }^{11}$ J. Lettieri, V. Vaithyanathan, S. Eah, J. Stephens, V. Sih, D. Awschalom, J. Levy, and D. Schlom, Appl. Phys. Lett. 83, 975 (2003).

${ }^{12}$ A. Swartz, J. Ciraldo, J. Wong, Y. Li, W. Han, T. Lin, S. Mack, J. Shi, D. Awschalom, and R. Kawakami, Appl. Phys. Lett. 97, 112509 (2010).

${ }^{13}$ C. Caspers, M. Müller, A. X. Gray, A. M. Kaiser, A. Gloskovskii, C. S. Fadley, W. Drube, and C. M. Schneider, Phys. Rev. B 84, 205217 (2011).

${ }^{14}$ D. Li, Y. Haga, H. Shida, T. Suzuki, Y. Kwon, and G. Kido, J. Phys.: Condens. Matter 9, 10777 (1997).

${ }^{15}$ C.-G. Duan, R. Sabirianov, W. Mei, P. Dowben, S. Jaswal, and E. Tsymbal, J. Phys.: Condens. Matter 19, 315220 (2007).

${ }^{16}$ W. Horne, P. Strange, W. Temmerman, Z. Szotek, A. Svane, and H. Winder, J. Phys.: Condens. Matter 16, 5061 (2004).

${ }^{17}$ C. Aerts, P. Strange, M. Horne, W. Temmerman, Z. Szotek, and A. Svane, Phys. Rev. B 69, 045115 (2004).

${ }^{18}$ M. Johannes and W. Pickett, Physical Review B. 72, 195116 (2005).

${ }^{19}$ B. Ruck, H. Trodahl, J. Richter, J. Cezar, F. Wilhelm, A. Rogalev, V. Antanov, B. Le, and C. Meyer, Phys. Rev. B. 83, 174404 (2011).

${ }^{20}$ S. Altendorf, A. Efimenko, V. Oliana, H. Kierspel, A. Rata, and L. Tjeng, Phys. Rev. B 84, 155442 (2011).

${ }^{21}$ P. Steeneken, New Light on EuO Films: Preparation, Transport, Magnetism and Spectroscopy of a Ferromagnetic Semiconductor Films: Preparation, Transport, Magnetism and Spectroscopy of a Ferromagnetic Semiconductor, Ph.D. thesis, University of Groningen (2002).

${ }^{22}$ R. Ulbricht, A. Schmehl, T. Heeg, J. Schubert, and D. Schlom, Appl. Phys. Lett. 93, 102105 (2008).

${ }^{23}$ R. Sutarto, S. G. Altendorf, B. Coloru, M. M. Sala, T. Haupricht, C. F. Chang, Z. Hu, C. Schuessler-Langeheine, N. Hollmann, H. Kierspel, H. H. Hsieh, H. J. Lin, C. T. Chen, and L. H. Tjeng, Phys. Rev. B 79, 205318 (2009).

${ }^{24}$ S. G. Altendorf, A. Efimenko, V. Oliana, H. Kierspel, A. D. Rata, and L. H. Tjeng, Phys. Rev. B 84, 155442 (2011).

${ }^{25}$ C. Caspers, M. Mller, A. X. Gray, A. M. Kaiser, A. Gloskovskii, C. S. Fadley, W. Drube, and C. M. Schneider, physica status solidi (RRL) Rapid Research Letters 5, 441 (2011).

${ }^{26}$ I. Langmuir, J. Am. Chem. Soc. 38, 2221 (1916).

${ }^{27}$ F. Voogt, P. Smulders, G. Wijnja, L. Niesen, T. Fujii, M. James, and T. Hibma, Phys. Rev. B 63, 125409 (2001).

${ }^{28}$ O. Rogojanu, Stabilizing CrO by Epitaxial Growth, Ph.D. thesis, University of Groningen (2002).

${ }^{29}$ I. Elfimov, A. Rusydi, S. Csiszar, Z. Hu, H. Hsieh, H. Lin, C. Chen, R. Liang, and G. Sawatzky, Phys. Rev. Lett. 98, 137202 (2007).

${ }^{30}$ R. Sutarto, S. G. Altendorf, B. Coloru, M. M. Sala, T. Haupricht, C. F. Chang, Z. Hu, C. Schuessler-Langeheine, N. Hollmann, H. Kierspel, J. A. Mydosh, H. H. Hsieh, C. T. Chen, and L. H. Tjeng, Phys. Rev. B 80, 085308 (2009).

${ }^{31}$ J. H. Scofield, J. Elect. Spect. Rel. Phenom. 8, 129 (1976). 